\title{
Deciphering neural heterogeneity through cell lineage tracing
}

\author{
María Figueres-Oñate $^{1,2} \cdot$ Rebeca Sánchez-González $^{1} \cdot$ Laura López-Mascaraque $^{1}$ D
}

Received: 30 July 2020 / Revised: 10 October 2020 / Accepted: 20 October 2020 / Published online: 5 November 2020 (c) The Author(s) 2020

\begin{abstract}
Understanding how an adult brain reaches an appropriate size and cell composition from a pool of progenitors that proliferates and differentiates is a key question in Developmental Neurobiology. Not only the control of final size but also, the proper arrangement of cells of different embryonic origins is fundamental in this process. Each neural progenitor has to produce a precise number of sibling cells that establish clones, and all these clones will come together to form the functional adult nervous system. Lineage cell tracing is a complex and challenging process that aims to reconstruct the offspring that arise from a single progenitor cell. This tracing can be achieved through strategies based on genetically modified organisms, using either genetic tracers, transfected viral vectors or DNA constructs, and even single-cell sequencing. Combining different reporter proteins and the use of transgenic mice revolutionized clonal analysis more than a decade ago and now, the availability of novel genome editing tools and single-cell sequencing techniques has vastly improved the capacity of lineage tracing to decipher progenitor potential. This review brings together the strategies used to study cell lineages in the brain and the role they have played in our understanding of the functional clonal relationships among neural cells. In addition, future perspectives regarding the study of cell heterogeneity and the ontogeny of different cell lineages will also be addressed.
\end{abstract}

Keywords Clonal analysis $\cdot$ Neural stem cell $\cdot$ Progenitor potential $\cdot$ Cell progeny $\cdot$ Ontogeny $\cdot$ Cell heterogeneity

\section{Introduction}

One fundamental issue in Neuroscience is how the different lineages in the brain are established and what contributions sibling cells make to the nervous system and how they influence its behavior. The current belief is that there is large cell heterogeneity in the adult brain, raising the question as to how these different cell types are generated during development. However, a fundamental question is whether this heterogeneity is ontogenically determined and if so, what are the physiological implications of this? Thus, lineage tracing has developed from the need to pursue all the progeny of specific neural progenitor cells (NPCs) to determine how complete neural networks are built and the contribution of specific progenitors to these networks.

Laura López-Mascaraque

mascaraque@cajal.csic.es

1 Department of Molecular, Cellular and Development Neurobiology, Instituto Cajal-CSIC, 28002 Madrid, Spain

2 Present Address: Max Planck Research Unit for Neurogenetics, 60438 Frankfurt am Main, Germany

\section{Neural stem cells potential and heterogeneity}

Neural stem cells (NSCs) are cells that self-renew and that can produce all the lineages present in the adult brain [1]. Thus, the cell diversity in the brain emerges as the progeny of NSCs progress into lineage-restricted NPCs, more committed cell populations with a more limited differentiation and proliferation potential [2]. The transition to a specific lineage and the consequent loss of potential takes place through symmetric or asymmetric cell divisions. Symmetric divisions amplify the pool of progenitors, generating two identical siblings, whereas asymmetric divisions generate two different daughter cells one of which at least will be more committed to a certain lineage (Fig. 1a).

At early embryonic stages, the principal neural lineages are specified in the neural plate, a defined region of the ectoderm. In parallel, cells of other origins colonize the prospective brain, such as microglia (mesoderm) and blood vessel cells (endoderm). Initially, NSCs known as neuroepithelial cells (NECs) undergo symmetric cell divisions to amplify their pool and prior to generating bipolar radial glial cells (RGCs) [3]. These RGCs produce all the major cell types in the brain and they are often considered the NSCs of the 
A

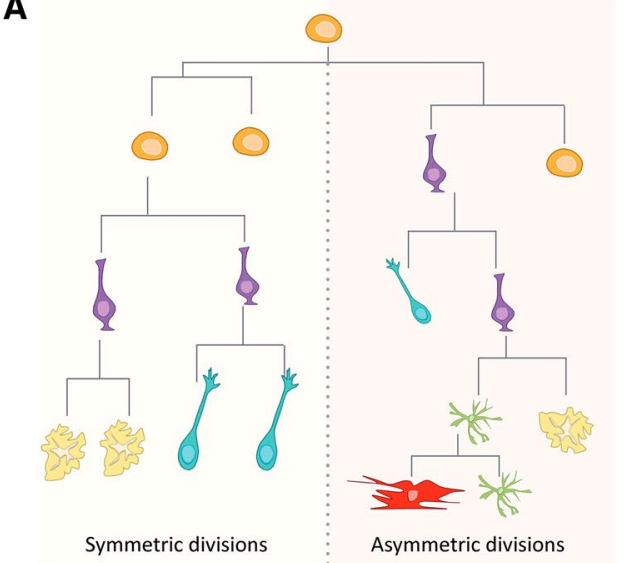

B

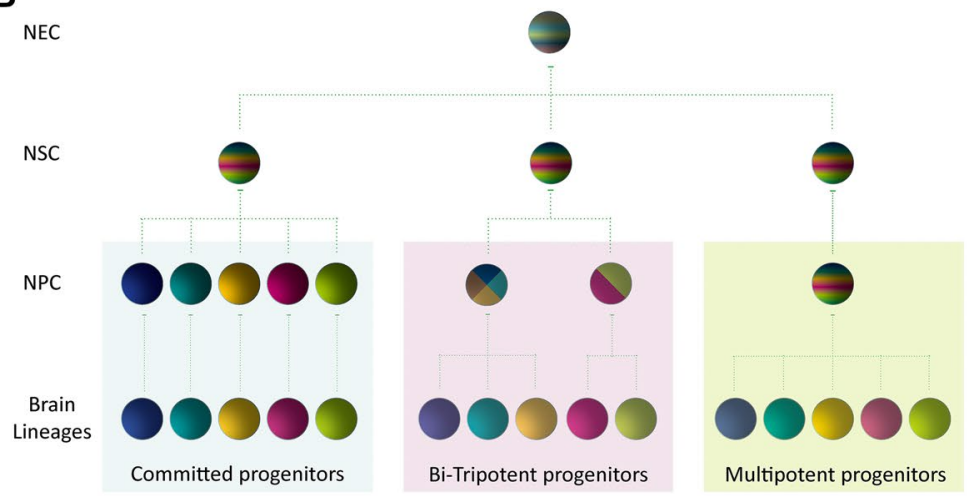

C

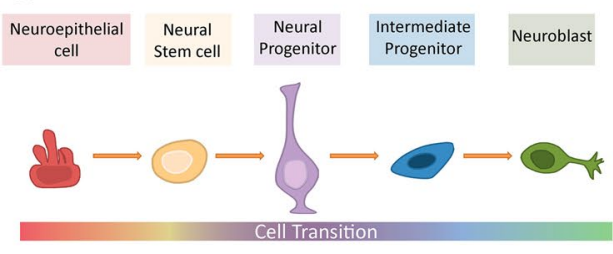

D

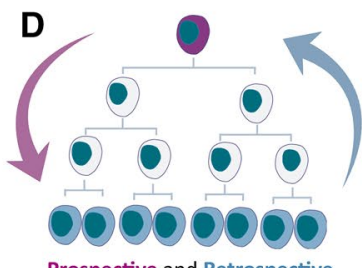

Prospective and Retrospective analysis

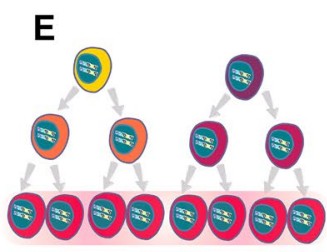

Lumping error

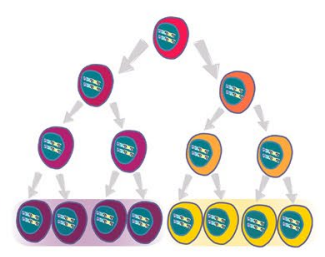

Splitting error
Fig. 1 a Lineage specification of stem cells throughout development occurs through symmetric and asymmetric divisions. b Scheme of the different progenitor potentials of the neuroepithelial cells (NECs) that may coexist in the developing brain. Committed progenitor cells can only give rise to one neural cell type, whereas multipotent progenitors can contribute to all lineages. c Graphical representation of the differentiation of NECs to neuroblasts. The gradual maturation of these cells is reflected in the morphological and molecular changes accompanying their lineage transitions. d Scheme of the prospective (pink) and retrospective (blue) reconstruction lineage approaches. Prospective lineage tracing targets specific progenitors to trace their progeny, whereas retrospective analyses reconstruct the lineage tree from the descendants to the progenitor. e Lumping errors are the result of considering non-clonally related cells as part of the same clone, whereas splitting errors take place when sibling cells are considered as independent clones due to methodological shortcomings. $N E C$ neuroepithelial cell, NSC neural stem cell, NPC neural progenitor cell developing brain [4]. RGCs can either proliferate symmetrically to maintain their pool or asymmetrically to generate intermediate progenitor cells (IPCs). RGCs or IPCs will then divide to generate neuroblasts, post-mitotic cells that are committed to generate mature neurons, or glial cells such as oligodendrocytes, astrocytes or NG2 cells [5]. RGCs form a complex cell population that displays region-specific gene expression in the developing nervous system [6]. NSCs are mainly located in the sub-ventricular zone (SVZ), a neurogenic niche that covers different microdomains of the ventricular embryonic walls comprising the pallium, subpallium and septum [7]. The neurogenic niche is considered as a heterogeneous pool of cells, with multiple progenitor types displaying either stem cell attributes or more restricted fates [8]. For example, multipotent NSCs that are capable of giving rise to all brain lineages may exist alongside tri- or bi-potent progenitors that have a more restricted potential [9-11]. NPCs that will contribute only one neural cell type could also be present in these neurogenic niches [12, 13] (Fig. 1b). In the brain, cell specification commences with neurogenesis, whereby neuroblasts give rise to the new neurons. Thereafter, astrogenesis occurs and ultimately, oligodendrogenesis commences that continues throughout perinatal stages [14]. This progression is regulated by intrinsic changes in gene expression but also, it is determined by interactions with environmental and developmental cues. Furthermore, the switch from neurogenesis to gliogenesis is driven by competition between downstream transcription factors and growth factor signaling [15, 16]. Different patterning genes are responsible for boundary formation, events that can determine the identity and fate of the NPCs in diverse domains along the lining of the ventricular surface [3].

New approaches in single-cell transcriptome technology have provided novel data regarding cell heterogeneity, addressing the varied gene expression in different brain populations. Specifically, a compilation of molecular markers has been described in the neurogenic niche, expressed in either active or quiescent NPCs under physiological conditions and after brain insult [17-19]. In these studies, cells from the SVZ have been identified based on the expression of particular antigenic markers. Thus, the identity and 
dynamics of NPCs have been assessed by considering the whole population of ventricular progenitors that express a specific combination of different markers. However, cells expressing selected markers but that are isolated from different areas display distinct self-renewal and differentiation capacities, and they have a distinctive gene expression profile [20]. Thus, no single molecular marker can unambiguously define separate populations. Lineage transition between NPCs and their progeny is achieved by gradual cell maturation, and thus, the expression of the same molecular markers in diverse cell types may overlap at distinct moments in their maturation (Fig. 1c). In the adult neurogenic niche of the SVZ, NSCs have classically been defined as GFAP (Glial Fibrillary Acidic Protein) expressing cells with a particular morphology [21], although this protein is also expressed by the surrounding astrocytes [22]. Furthermore, CD133 (or prominin1) is a transmembrane glycoprotein expressed by the cilia of the adult NSCs in the SVZ [23], and it is also a molecular marker expressed in ependymal cells populating the same surface [24]. Nestin has been considered to be a typical marker to identify NSCs, although it is still expressed in the IPC population [25]. Another molecular marker used to identify embryonic and adult NSC populations is CD24, which is also expressed in some neuroblast populations, albeit at different levels [26]. Thus, cell diversity studies should be based on the potential of single progenitors rather than that of NPCs pools, requiring finer analyses at the individual cell level.

Together, defining NPC populations using specific markers has some limitations, as it is often impossible to precisely define these complex and heterogeneous cell populations solely in this manner. Accordingly, clonal analysis and lineage tracing has become crucial to further understand the biology of NSCs and their lineage progression [27, 28].

\section{The onset of lineage tracing}

Due to the large heterogeneity within the neural progenitor pool, clonal analysis is particularly important when studying stem cell biology. This concept arose at the end of the nineteenth century with the studies of Whitman and collaborators on leech embryos [29], and it is still the subject of intense study today. Cell lineage tracing facilitates the definition of ontogeny, fate and cell behavior in specific tissues or organisms. In the brain, lineage tracing allows the cell progeny of a single neural progenitor to be identified and tracked, and the clonal relationships among co-habiting cells to be defined in the adult. Labeling all the progeny of a specific progenitor or cell population may reveal specific patterns of progenitor proliferation, migration and differentiation. Different prospective and retrospective methodologies have been developed to study cell lineages in the brain (Fig. 1d). Prospective lineage tracing strategies require an initial population of interest to be targeted that will be followed over time, whereas retrospective analysis aims to reconstruct lineage trees from the descendants based on a non-bias selection of the progenitors. The principal drawback when designing clonal methods are the potential lumping and splitting errors (Fig. 1e). Lumping errors arise from considering cells generated from different progenitors as part of the same clone. By contrast, cells that are part of the same lineage could be treated as non-sibling cells due to methodological issues, leading to splitting errors.

Some of the first complete lineage tracing studies were achieved by direct microscope observation of lineage progression using either organisms with a small number of cells, or by isolating NPCs and tracking their divisions in vitro [30]. A ground breaking achievement in the field was the tracing of the entire lineage tree of the nematode C. elegans [31] by time-lapse microscopy. However, direct observation in vivo is not usually viable due to the opacity of the tissue or the aim of studying a larger organism. Nevertheless, direct observation of embryonic development in vivo through an intravital window can be combined with different cell labeling approaches, and this has permitted the monitoring, manipulation and live imaging of mouse embryos [32]. The incorporation of non-toxic chromogens into living cells, referred to as vital dyes, was one of the first methods used to visualize cells over time. The principal advantage of fluorescent vital dyes is that they are easily administered in vivo and they do not require post-processing to be visualized. Among the initial fluorescent dyes widely used were the fluorescent retrograde markers like Fast Blue (a cytoplasmic marker) and Diamidino Yellow (a nuclear marker). These dyes not only produced retrograde labeling of groups of cells but they could also be injected simultaneously to detect double labeled cells [33]. In addition, lipophilic carbocyanine fluorescent dyes like DiI and DiO have been used for anterograde and retrograde neuronal tracing in vivo, and in fixed tissue [34,35], offering a detailed view of the cell's morphology. The labeling of proliferative cells by incorporating a nucleoside analog like 5-Bromo2 'deoxyuridine (BrdU) can be also used to study cell lineage and fate potential $[36,37]$ However, a drawback in these approaches is the dilution of the tracer, triggering the consecutive loss of labeling that is most evident in actively proliferating cells [38].

The discovery of the green fluorescent protein (GFP) [39] and of $\beta$-galactosidase encoded by the $E$. coli LacZ gene [40] revolutionized lineage tracing, taking over from the use of vital dyes. Reporter genes encoding these proteins were introduced to target cells by lipofection [41] or electroporation [42], and viral particles carrying these reporters could infect cells, integrating the desired recombinant DNA into the host genome to allow cell tracing [43]. Retroviruses have been used widely to trace cell lineages [44-46]. The reporter 
genes are introduced via retroviral vectors that integrate into the genome of dividing target cells and they are consequently transferred to all their progeny. The first viral tracing methods focused on targeting a limited number of sparse progenitor cells to ensure that the labeled cells populating the same area would pertain to the same clone. Serial dilution of the viral particles can be used to target fewer isolated progenitors. However, an increment of the number of traceable lineages and a stronger lineage analysis was obtained using retroviral libraries that include DNA barcoding [47]. These barcodes can be read after cell sorting or laser dissection, permitting a reconstruction of the lineages. Nevertheless, the use of retroviral labeling can be compromised by epigenetic silencing and the inability to transfect quiescent or non-mitotic cells [48].

Tracking cells from their progenitors to their final destination and fate has been made possible through the development of some important genetic tools that have made permanent cell labeling feasible. In particular, the Cre-LoxP [49] and Flp-FRT [50] recombination systems represented an important advance to study cell progeny. The incorporation of a tamoxifen-inducible version of this technology in transgenic mouse lines expanded the possibilities of undertaking cellular studies in genetically modified organisms (GMOs) [51]. Transgenic mouse lines that are susceptible to inducible-Cre recombination under the control of specific promoters can drive the expression of a fluorescent reporter (FR) to fate map neural progenitors in vivo [52]. This has been achieved by administering low doses of tamoxifen and depending on the lineage of interest, transgenic mice encoding different FRs under the control of specific promoters have been generated, exhibiting particular advantages and disadvantages [53].
In addition, early lineage tracing studies used chimeric mice generated from tetraparental embryos enabled the origin of many structures in the body to be determined [54]. Moreover, transplanting labeled cells from GMOs into wild type animals has also been used to trace cell lineages [55, 56], although one shortcoming of this approach is that the grafted cells may not behave as they do under physiological conditions [57].

\section{Multicolor lineage tracing}

The employment of GMOs and the incremental growth of reporters led to the idea of combining different fluorescent proteins to track sibling cells (Fig. 2). Initially, a few fluorescent variants of GFP were combined to stain neurons individually [58] but undoubtedly, the true revolution in multicolor lineage methods commenced with the appearance of the Brainbow technology [59]. Brainbow transgenic mice undergo stochastic recombination of up to four FRs that are driven by the Cre-LoxP system, giving rise to multicolor mosaics in which single cells can be easily identified. Modified versions of this methodology are still being developed $[60,61]$ and they produce vivid images, that allow an extremely detailed visualization of the morphologies of individual labeled cells. Hence, this is a very effective method for cell mapping but not for lineage tracing. However, this technology has had a tremendous impact on the field of lineage tracing, leading to a new wave of methods in which the stochastic combination of FRs is used to generate unique barcodes in NSCs that can be inherited by all their progeny. This method was originally designed for mice, although the combinatorial use of fluorescent proteins has been remodeled to fate mapping in Drosophila

\section{Multicolor lineage methods}

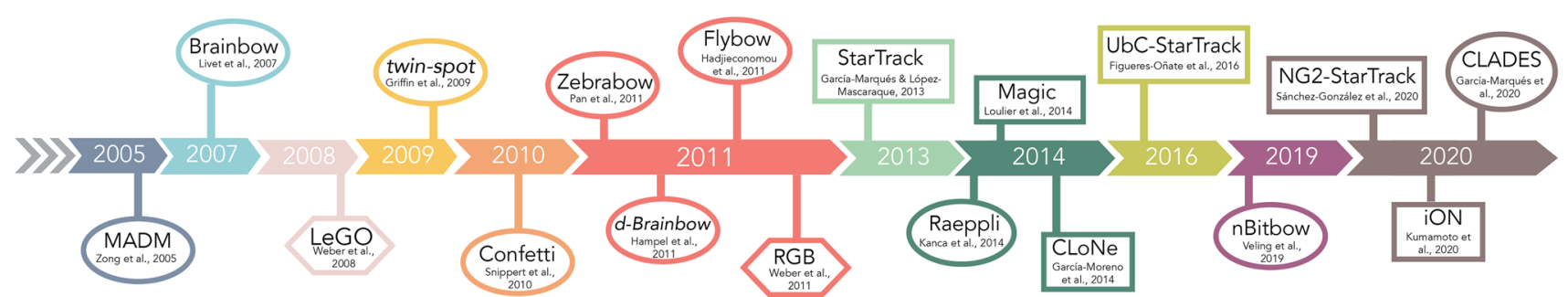

Genetically modified organisms

Viral vectors

DNA constructs

Fig. 2 Timeline of multicolor lineage methods. Diagram of the most relevant approaches using combinations of fluorescent reporter proteins for fate mapping. The different strategies are represented by a circle for genetically modified organisms like mice, zebrafish or Dros- ophila. A hexagon shows the strategies based on recombinant viral particles, and the square represents those approaches involving the use of DNA constructs encoding the different fluorescent reporters 
melanogaster (Flybow, d-Brainbow, Raeppli) [62-64] and Zebrafish (Zebrabow) [65, 66]. The nuclear expression of more than one fluorophore per cell has generated new modifications of the Brainbow-like technique in Drosophila (nBitbow) [67]. Other clonal methods isolate recombination in stochastic cells involving different transgenic lines, such as the mosaic analysis with double markers (MADM) [68, 69], and this technology has also been modified to D. melanogaster (twin-spot) [70]. The principal drawback of these techniques, besides the requirement for GMOs, is the small range of combinations to accurately define daughter cells, and the susceptibility to clonal splitting or lumping errors. Moreover, visualizing the fluorescent signal in some of these transgenic mice requires immunostaining [28], an important limitation due to the lack of antibodies to specifically recognize these FRs. Thus, to extend multicolor lineage tracing to different organisms or even to in vitro assays, recombinant viral particles or DNA plasmids have been designed. Recombinant lentiviruses encoding different FRs have been generated to contribute to the multicolor mosaic for lineage tracing (LeGo) [71, 72]. New approaches involve recombinant DNA constructs that encode different FRs, and that can be transfected into the cells of interest, have allowed all the progeny of single cells to be traced (StarTrack, CLONE, MAGIC, iON) [73-76]. To resolve the timing of the birth of each cell within the same clone, lineage progression could be determined post hoc by the expression of a predetermined sequence of fluorophores in sibling cells in Drosophila (CLADES) [77]. These methods are based on transposable elements that are integrated into the genome, allowing the stable inheritance of the same barcode by all cell progeny, and avoiding plasmid loss as a consequence of cell division. The combinatorial use of integrable FRs does not require post-processing to visualize the signals due to the bright and stable expression of these proteins, thereby representing an accessible and convenient technique to use in vivo. Moreover, injection of the reporter proteins in vitro or in vivo facilitates the targeting of progenitor cells at a single-cell level, avoiding the need to produce GMOs. New advances in microscopy have produced progress in multicolor lineage tracing [78], expanding the possibility of performing lineage tracing in any organism and lineage, and enabling studies of cell heterogeneity.

Our laboratory has developed a stochastic clonal analysis method called StarTrack, which allows the progeny of single cells to be traced and analyzed thereafter. The StarTrack methodology was an attempt to develop a genetic in vivo lineage-tracing method that could track all the neural progeny of individual GFAP cells [73]. It is based on the transfection of cells (by electroporation) with a combination of recombinant DNAs encoding six different FRs that are expressed in different cell compartments (cytoplasm or cell nucleus). This produces inheritable marks that permit the long-term in vivo tracking of the different neural cells generated during embryonic development to their final fate in the adult brain. Due to the use of an ubiquitous promoter, the UbCStarTrack methodology enables the progeny of embryonic and postnatal neural progenitors to be tracked in mice, irrespective of their fate [79]. Recently, to specifically track the descendants of NG2 progenitors, the promoter of either the transposase or transposon constructs was adapted accordingly $[80,81]$. Novel methods based on vector integration show high efficiency in terms of stable integration and the transmission of unique fingerprints to their descendants, which can be followed through several cell divisions and along different lineages through postnatal or adult stages. Furthermore, these strategies can be extrapolated to perform clonal and functional analyses in different animal models or in vitro assays. The increase in the number of FRs expressed in different compartments augments the number of possible combinations available in these integrable multicolor methods. For example, the use of six different fluorophores in two different cell compartments (e.g., nuclear and cytoplasmic) leads to a total of 4095 possible color codes. In addition, the number of copies of each reporter could be resolved by analyzing the intensity of fluorescence in each cell [79], helping to minimize possible splitting and lumping errors.

In summary, multicolor image-based lineage tracing methods are among the most reliable methods to define lineage trees. By combining them with state-of-the-art approaches like cell type-specific optogenetic manipulations, single-cell transcriptomic analysis, cell ablation, live-cell imaging, patch-clamp recordings, two-photon microscopy, in vivo and brain slice preparations, they may help us to better understand how lineages are derived in the brain. In addition, they provide a more functional readout of the specific characteristics of clonally related cells derived from the same NPC, adding information regarding their spatial relationships.

\section{Single-cell sequencing and CRISPR to address cell lineage tracing}

Next-generation sequencing (NGS) provides us with a powerful tool to sequence the whole genome and it opens a window to complete the study of whole organisms, elucidating their genomic, transcriptomic, epigenomic and proteomic profiles. NGS contributes to lineage tracing through both whole genome sequencing and whole-exome sequencing. It has revealed somatic mutations that accumulate in cells after replication that can be traced to define lineage progression [82]. These analyses focused on individual cells, revealing the intricate cell heterogeneity at the single-cell level. Single-cell RNA sequencing (scRNA-seq) is one of the most powerful tools to identify patterns of cell expression and intrinsic molecular profiles. However, single-cell 
transcriptomic analysis lacks information regarding ontogenic and spatial tracing. Genomic barcoding using viral libraries, followed by the isolation and sequencing of single cells, has facilitated the fate mapping of neural cells, helping to understand their ontogeny and their lineage potential, and complementing classical barcoding methods [83, 84]. Similarly, the combination of classic barcoding techniques and scRNA-seq could help understand the complex biological systems underlying physiological events and pathological conditions.

Several other strategies have been introduced in recent years, including the implementation of CRISPR technology to drive genome editing and DNA targeting (Fig. 3). The CRISPR/Cas9 was used to perform cell lineage tracing, in theory enabling the entire organism to be reconstructed at the single-cell level using RNA or DNA sequencing.
MEMOIR (mutagenesis with optical in situ readout) was the first method based on CRISPR technology that produced dynamic cell records and lineage reconstruction, combining barcoded recording elements (scratchpad) altered by CRISPR-Cas9 genome editing and in situ readouts by seqFISH RNA imaging [85] (Fig. 3a). The limitation of this system is the lower capacity to detect edited mutations relative to those that used scRNA-seq. One of the first studies to implement CRISPR for phylogenetic analyses in mice was MARC1 (mouse for actively recording cells). These mice can accumulate genomic mutations that can be used to reconstruct the lineage tree of cells based on the homing guide RNA (hgRNA) and CRISPR/Cas9 nuclease [86]. In addition, zebrafish has been the animal model used to develop LINNAEUS (Lineage tracing by Nuclease-Activated Editing of Ubiquitous Sequences) [87], in which
Fig. 3 Timeline of the use of CRISPR/Cas9 for lineage tracing. a In situ mutagenesis involved the accumulation of multiple integration target sites (called barcoded scratchpads) visualized by smFISH.

This strategy is a good tool to address the lineage dynamics of a cell population. $\mathbf{b}$ In vivo lineage mapping using CRISPR/ Cas9 is based on multiple integration/deletion at a target site that allows the lineage trees of the different cells to be reconstructed taking into account their genomic tags (yellow cassettes). c Going further, the inducible Cas9 activity (blue circle) and the incorporation of transcriptomic analysis improves these mouse models, enabling cell lineages and fate mapping to be analyzed at the single-cell level

\section{A In situ mutagenesis}

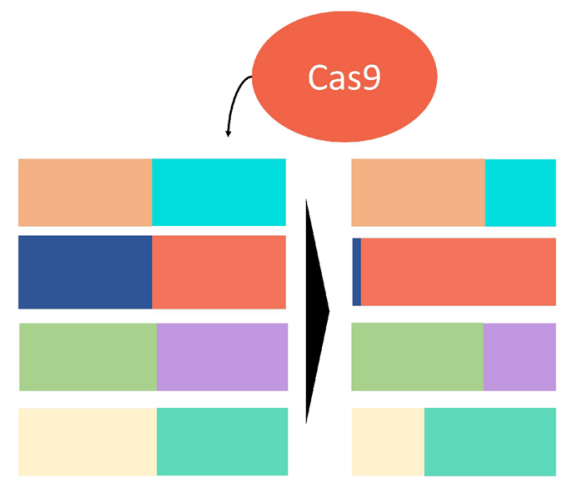

\section{Lineage cell dynamics}

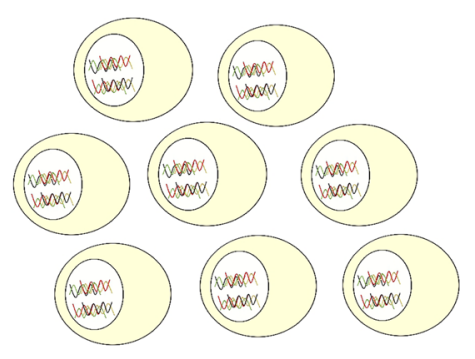

B In vivo lineage mapping

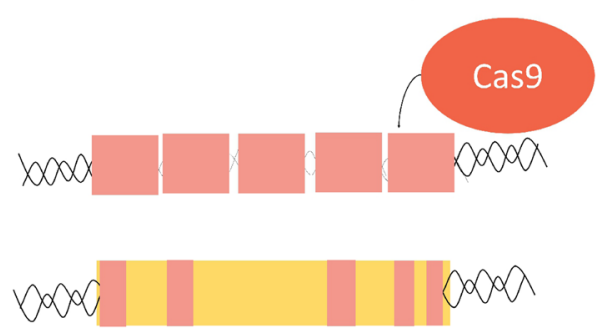

Lineage tree

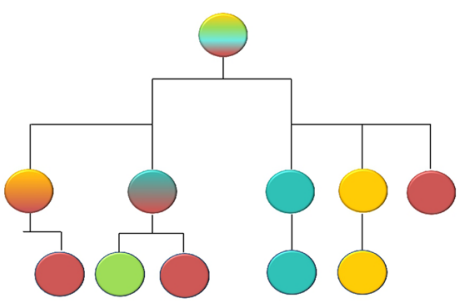

C Genome-editing barcoding

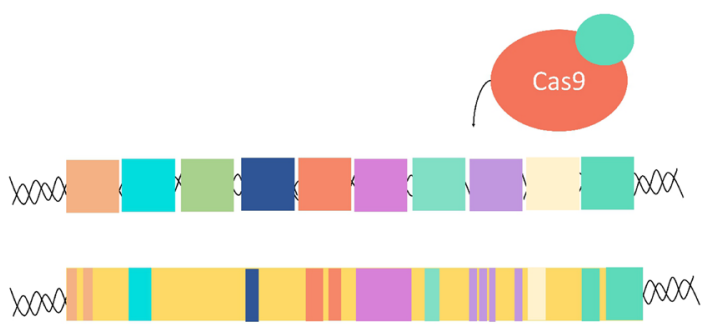

Differential gene analysis

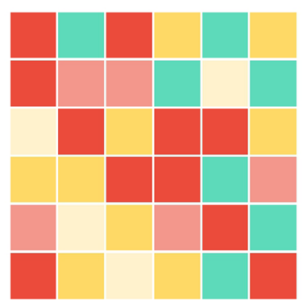


lineage trees of zebrafish larvae and adult cells were reconstructed by combining multiple genomic mutations and transcriptome profiling (Fig. 3b). However, the first study to infer a large-scale lineage potential was called GESTALT (genome editing of synthetic target array for lineage tracing), revealing lineage relationships during germ layer patterning in zebrafish. GESTALT employed embryos carrying an array of ten different targets that created an inheritable barcode permitting cell lineage trees to be reconstructed [88]. Nevertheless, this system was not able to distinguish the spatial location of different cell types and it was restricted to early developmental studies. Subsequently, scGESTALT was developed to improve this system, combining CRISPR/ Cas9 barcode editing for large-scale lineage tracing with cell characterization using transcriptomic analyses [89]. This new approach enabled cell editing to be performed at multiple points and at later developmental stages, and accordingly it has been established as a powerful tool that enables simultaneous lineage tracing and cell sequencing in vivo [90]. These approaches permit the single-cell clonal dynamics and the transcriptomic profile of cells to be studied (Fig. 3c). Importantly, the clonally related cells located in the same area presented similarities in their transcriptomic profile. More recently, a new mouse line has been obtained using CRISPR/Cas9 genome editing based on lineage tracing, called CARLIN (CRISPR array repair lineage tracing: [91]). This revolutionary tool can be used to modify and detect DNA sequences in single cells line, and it could even record the history of specific stimuli, including the lineage related effects of exposure to external stimuli, stress or pathogens. One of the advantages of CARLIN is the inducible activity of Cas 9 to generate barcodes at embryonic and adult stages. In conclusion, several models of CRISPR/Cas9 barcoding have shown this is a powerful tool to study fate mapping. However, cell loss or weak RNA expression are among the technical problems encountered when using these methods [92]. Nevertheless, the evolution of CRISPR-Cas9 technology along the new sequencing approaches offers very useful approaches to define fate maps in complex organisms.

\section{Insights into the lineage tracing of neural cells from clonal methodology}

Several methods have been proposed to examine lineage progression in different biological models in vivo or in vitro. However, their use to determine the relationships of lineages usually requires an arduous analysis. Some approaches for lineage tracing in the brain focus on the sparse labeling of cell clones to ensure the lineage connection among cells that are situated intimately in the brain (Fig. 4a). The injection of diluted viral particles to trace a minimal number of progenitors facilitates the study of the integration of neural clones into different cortical systems
[93-95]. Moreover, administering low doses of tamoxifen to inducible Cre-LoxP GMOs favors the characterization of NSC potential [96]. Random Cre-LoxP recombination in sparse progenitor cells during development reveals a common origin of pyramidal neurons and astrocytes [97]. However, these methods have some drawbacks, such as the ambiguous labeling of single NPCs, the impossibility of tracing lineages that undergo diverse migratory pathways and the difficulty for inter-clonal studies. Moreover, the independent basal recombinase activity of inducible Cre lines may interfere with such tamoxifen-based lineage tracing methods [98].

Other clonal methods rely on the recombination of few fluorophores for lineage tracing (Fig. 4b), such as mosaic analysis with dual markers that have been used to determine important features of NPC potential and lineage progression in vivo $[27,99,100]$. These approaches help characterize sibling cells at a single-cell level, although only a few codes are generated for unequivocal cell identification and sparse labeling is still required.

More recently, the use of fingerprints to accurately target a single NPC and that can be inherited by their entire progeny has revolutionized the field (Fig. 4c). With these methods a larger number of clones can be assessed, allowing inter- and intra-clonal relationships to be studied, as well as identifying clonal relationships within long-distance migratory cell populations, such as adult generated interneurons [11]. Progenitor barcoding can be achieved by expressing multiple FRs, retroviral libraries or with genetic tags, such as those assembled by the CRISPR-Cas9 or Polilox system [101]. The huge improvement in sequencing technologies and genome editing tools over recent years has enriched the lineage tracing field. These techniques enable phylogenetic lineage trees to be created and linked to transcriptomic information at a single-cell level. The developing central nervous system (CNS) contains many spatially segregated germinal zones, with progenitors that generate distinct cell types. The fate of embryonic progenitors lining either the ventricular surface or the ependymal layer of the developing brain can be traced, and post-natal or adult NPCs in the brain can be also included in the lineage tracing analysis. Clonal methods have demonstrated that these progenitors may be committed to a specific cell lineage or that they may give rise to more lineages. RGCs are considered the major progenitor cell type throughout the CNS. However, new sub-types of NPCs occupy the SVZ, such as apical or basal RGCs, or apical and subapical IPCs [102]. Lineage tracing has shown that RGCs produce glial cells through IPCs or astrocytes by direct transformation [103, 104]. In addition, embryonic RGCs give rise to sibling ependymal cells and adult NSCs that remain in the lateral ventricles [105]. Postnatal NPCs have varied multipotentiality, which differs from that of restricted progenitors specified to only generate 

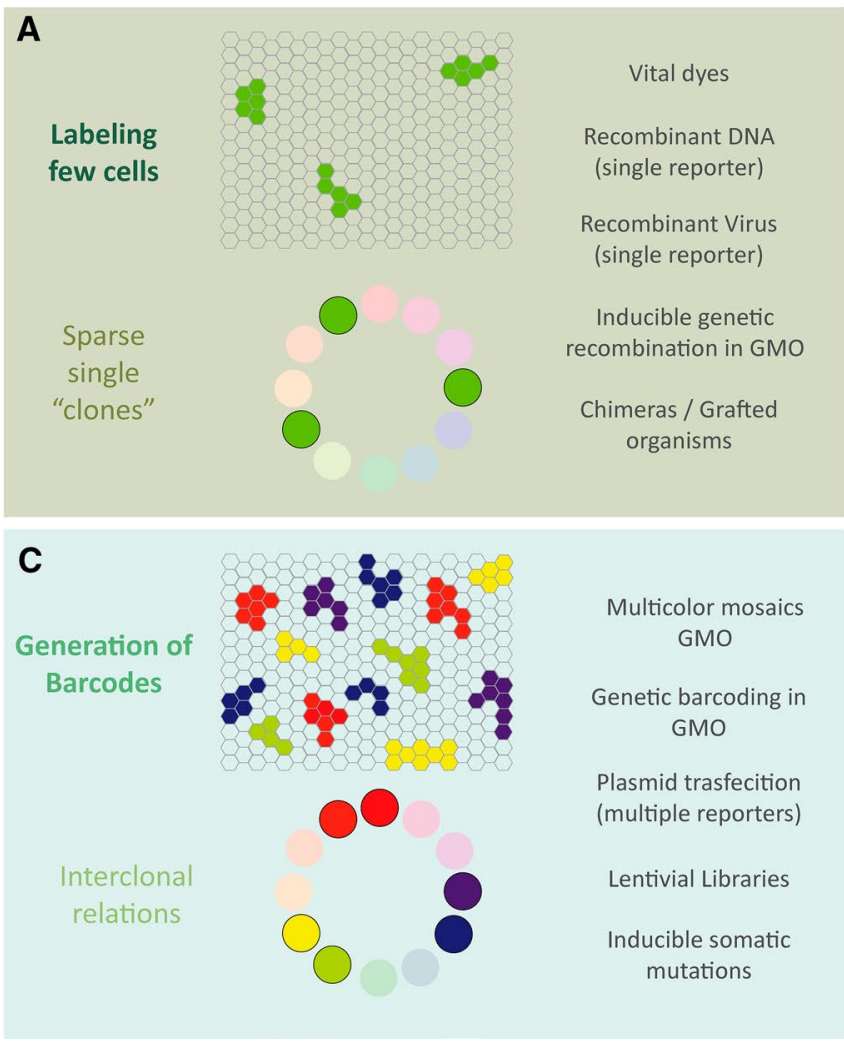

Fig. 4 Diagram of the different techniques and their improvements based on advances in lineage tracing. a The first lineage analyses were done by random targeting of progenitors in a manner that attempted to ensure the distant labeling of clones. b The incorporation of more than one reporter increased the number of combinations and improved the single-cell clone identity. c The assignment

a determined lineage, or of bi-potent NPCs that generate sibling cells of two different lineages [11].

Single-cell analyses has provided huge advantages when studying cell heterogeneity and cell dynamics in NSC populations. Transcriptomic approaches have recently shed new light on lineage progression and progenitor potential, and single-cell transcriptomic analysis has revealed important information about cell identity and the distinct expression patterns under different conditions that could be crucial to fine-tune lineage tracing approaches. Transcriptomic analyses have helped classify cells in terms of their specific transcriptomic expression. Several studies have shown the diversification of transcriptional profiles within neuronal [106, 107], astroglial [108, 109] and oligodendroglial populations [110, 111]. Recent approaches using in situ transcriptomic analyses reflect the strong specification and regionalized distribution of astrocytes within the pallial cortex, diverging from the classic six neuronal layered patterns [112]. In addition, bulk RNA-seq has provided important information about gene expression and the molecular differences between cells populating neocortical layers [113].
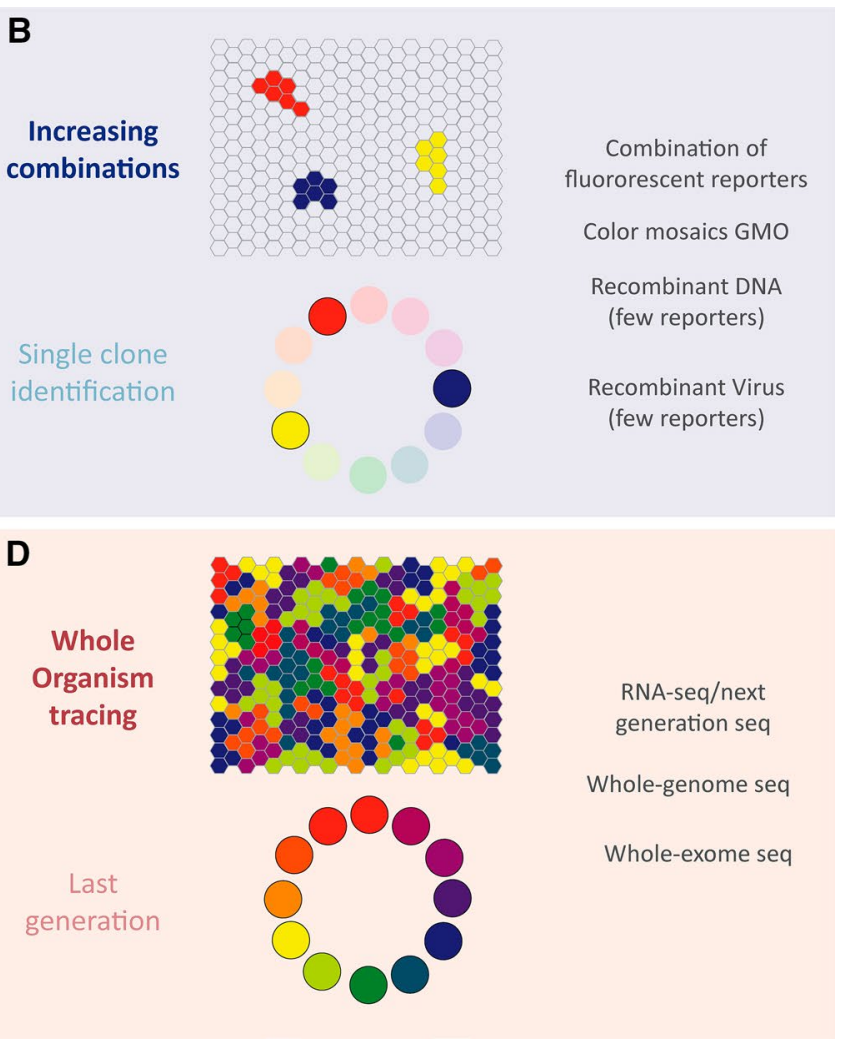

of unique fingerprints to targeted progenitors enabled sibling cells to be identified and their relationships due to the generation of a specific and stable barcode in the cells. d However, the new tools generated to decipher genomic information in an entire organism enable a wealth of important information to be drawn that helps to reconstruct the lineage trees of cells and their sibling connections

Furthermore, pseudotemporal alignment of the transcriptomic profiles of developing cerebral organoids gave insight into the maturation and differentiation stages associated with cell-type specification [114]. The regional identity of specific neuronal progenitors has also been described for glial lineages, where progenitors in different domains produce glial cells restricted to a specific region $[13,115]$, with broad variability in terms of their spatial and clonal organization [116]. Thus, it becomes crucial to determine how embryonic development influences cell fate heterogeneity.

In addition, deep genome sequencing has made it possible to trace the lineage of every single cell in a given organism (Fig. 4d). DNA replication that occurs before cell division produces somatic mutations that do not have phenotypic effects. However, these somatic DNA mutations accumulate in daughter cells and they provide important information that could be useful to reconstruct lineage trees $[117,118]$.

Lineage tracing techniques can also be applied in pathological situations to address the heterogeneity in terms of therapeutic responses or the different implications according to ontogeny. In this regard, clonal responses have been 
described in the progression of squamous carcinomas [119], sarcomas [120] and breast cancers [121]. In the CNS, glioblastoma cells engage in clonal communication based on cell-cell contact [122]. Alternatively, clonal expansion of the astrocyte lineage was evident following brain disease or insult, as seen for Huntington's disease [123], brain injury [124] or multiple sclerosis [125]. This clonal response could be explained by preferential connectivity of sibling astrocytes relative to their neighboring cells [126]. Therefore, fate mapping and cell fate potential mapping could provide important information about lineage trees and evolution, yet it could also shed light on important therapeutic issues or even, on the possible reprogramming of cells. One challenging issue will be to compile and compare all the lineage tracing data obtained through different clonal methodologies to obtain an overview of how lineages evolve [127]. All the emerging data, along with the improved molecular techniques and the developments in big data analysis, can overcome the current limitations to understand the tremendous heterogeneity among cell lineages.

In conclusion, DNA barcoding strategies and sequencing resources could help to consolidate cell lineage reconstructions and contribute to our understanding of cell dynamics, clonal expansion, and the behavior of specific cell lineages in normal conditions and disease. Moreover, this type of analysis may produce important advances in understanding the molecular events underlying lineage specification and the sculpting of progenitor potential.

Acknowledgements This work was supported by research Grants from MICINN (PID2019-105218RB-I00), MINECO (BFU2016-75207-R) and Fundación Ramón Areces (Ref. CIVP9A5928).

Open Access This article is licensed under a Creative Commons Attribution 4.0 International License, which permits use, sharing, adaptation, distribution and reproduction in any medium or format, as long as you give appropriate credit to the original author(s) and the source, provide a link to the Creative Commons licence, and indicate if changes were made. The images or other third party material in this article are included in the article's Creative Commons licence, unless indicated otherwise in a credit line to the material. If material is not included in the article's Creative Commons licence and your intended use is not permitted by statutory regulation or exceeds the permitted use, you will need to obtain permission directly from the copyright holder. To view a copy of this licence, visit http://creativecommons.org/licenses/by/4.0/.

\section{References}

1. Gage FH (2000) Mammalian neural stem cells. Science (80-) 287:1433-1438. https://doi.org/10.1126/science.287.5457.1433

2. Temple $S$ (2001) The development of neural stem cells. Nature 414:112-117. https://doi.org/10.1038/35102174

3. Rowitch DH, Kriegstein AR (2010) Developmental genetics of vertebrate glial-cell specification. Nature 468:214-222. https:// doi.org/10.1038/nature09611
4. Guo C, Eckler MJ, McKenna WL et al (2013) Fezf2 expression identifies a multipotent progenitor for neocortical projection neurons, astrocytes, and oligodendrocytes. Neuron 80:1167-1174. https://doi.org/10.1016/j.neuron.2013.09.037

5. Götz M, Huttner WB (2005) The cell biology of neurogenesis. Nat Rev Mol Cell Biol 6:777-788. https://doi.org/10.1038/nrm17 39

6. Kriegstein AR, Götz M (2003) Radial glia diversity: a matter of cell fate. Glia 43:37-43. https://doi.org/10.1002/glia.10250

7. Azim K, Fiorelli R, Zweifel S et al (2012) 3-Dimensional examination of the adult mouse subventricular zone reveals lineagespecific microdomains. PLoS ONE. https://doi.org/10.1371/journ al.pone. 0049087

8. Franco SJ, Müller U (2013) Shaping our minds: stem and progenitor cell diversity in the mammalian neocortex. Neuron 77:19-34. https://doi.org/10.1016/j.neuron.2012.12.022

9. Ortiz-Álvarez G, Daclin M, Shihavuddin A et al (2019) Adult neural stem cells and multiciliated ependymal cells share a common lineage regulated by the geminin family members. Neuron 102:159-172.e7. https://doi.org/10.1016/j.neuro n.2019.01.051

10. Parmigiani E, Leto K, Rolando C et al (2015) Heterogeneity and bipotency of astroglial-like cerebellar progenitors along the interneuron and glial lineages. J Neurosci 35:7388-7402. https ://doi.org/10.1523/JNEUROSCI.5255-14.2015

11. Figueres-Oñate M, Sánchez-Villalón M, Sánchez-González R, López-Mascaraque L (2019) Lineage tracing and cell potential of postnatal single progenitor cells in vivo. Stem Cell Reports 13:700-712. https://doi.org/10.1016/j.stemcr.2019.08.010

12. Ortega F, Gascón S, Masserdotti G et al (2013) Oligodendrogliogenic and neurogenic adult subependymal zone neural stem cells constitute distinct lineages and exhibit differential responsiveness to Wnt signalling. Nat Cell Biol 15:602-613. https://doi. org/10.1038/ncb2736

13. García-Marqués J, López-Mascaraque L (2016) Clonal mapping of astrocytes in the olfactory bulb and rostral migratory stream. Cereb Cortex. https://doi.org/10.1093/cercor/bhw071

14. Kriegstein A, Alvarez-Buylla A (2009) The glial nature of embryonic and adult neural stem cells. Annu Rev Neurosci 32:149-184. https://doi.org/10.1146/annurev.neuro.05150 8.135600

15. Sauvageot C, Stiles D (2002) Molecular mechanisms controlling cortical gliogenesis. Curr Opin Neurobiol 12:244-249. https:// doi.org/10.1016/S0959-4388(02)00322-7

16. Martynoga B, Drechsel D, Guillemot F et al (2012) Molecular control of neurogenesis: a view from the mammalian cerebral cortex molecular control of neurogenesis: aview from the mammalian cerebral cortex. Cold Spring Harb Perspect Biol 4:1-14. https://doi.org/10.1101/cshperspect.a008359

17. Codega P, Silva-Vargas V, Paul A et al (2014) Prospective identification and purification of quiescent adult neural stem cells from their in vivo niche. Neuron 82:545-559. https://doi. org/10.1016/j.neuron.2014.02.039

18. Beckervordersandforth R, Tripathi P, Ninkovic J et al (2010) In vivo fate mapping and expression analysis reveals molecular hallmarks of prospectively isolated adult neural stem cells. Cell Stem Cell 7:744-758. https://doi.org/10.1016/j.stem.2010.11.017

19. Llorens-Bobadilla E, Zhao S, Baser A et al (2015) Single-cell transcriptomics reveals a population of dormant neural stem cells that become activated upon brain injury. Cell Stem Cell 17:329-340. https://doi.org/10.1016/j.stem.2015.07.002

20. Pfenninger CV, Steinhoff C, Hertwig F, Nuber UA (2011) Prospectively isolated CD133/CD24-positive ependymal cells from the adult spinal cord and lateral ventricle wall differ in their long-term in vitro self-renewal and in vivo gene expression. Glia 59:68-81. https://doi.org/10.1002/glia.21077 
21. Imura T, Kornblum HI, Sofroniew MV (2003) The predominant neural stem cell isolated from postnatal and adult forebrain but not early embryonic forebrain expresses GFAP. J Neurosci 23:2824-2832

22. Nolte C, Matyash M, Pivneva T et al (2001) GFAP promotercontrolled EGFP-expressing transgenic mice: a tool to visualize astrocytes and astrogliosis in living brain tissue. Glia 33:72-86. https://doi.org/10.1002/1098-1136(20010101)33:1\%3c72::AIDGLIA1007\%3e3.0.CO;2-A

23. Mirzadeh Z, Merkle FT, Soriano-Navarro M et al (2008) Neural stem cells confer unique pinwheel architecture to the ventricular surface in neurogenic regions of the adult brain. Cell Stem Cell 3:265-278. https://doi.org/10.1016/j.stem.2008.07.004

24. Coskun V, Wu H, Blanchi B et al (2008) CD133+ neural stem cells in the ependyma of mammalian postnatal forebrain. Proc Natl Acad Sci U S A 105:1026-1031. https://doi.org/10.1073/ pnas.0710000105

25. Gengatharan A, Bammann RR, Saghatelyan A (2016) The role of astrocytes in the generation, migration, and integration of new neurons in the adult olfactory bulb. Front Neurosci 10:1-8. https://doi.org/10.3389/fnins.2016.00149

26. Pruszak J, Ludwig W, Blak A et al (2009) CD15, CD24, and CD29 define a surface biomarker code for neural lineage differentiation of stem cells. Stem Cells 27:2928-2940. https:// doi.org/10.1002/stem.211

27. Gao P, Postiglione MP, Krieger TG et al (2014) Deterministic progenitor behavior and unitary production of neurons in the neocortex. Cell 159:775-788. https://doi.org/10.1016/j. cell.2014.10.027

28. Calzolari F, Michel J, Baumgart EV et al (2015) Fast clonal expansion and limited neural stem cell self-renewal in the adult subependymal zone. Nat Neurosci 18:490-492. https://doi. org/10.1038/nn.3963

29. Whitman C (1887) A contribution to the history of germ layers in clepsine. J Morphol 1:105-182

30. Temple S (1989) Division and differentiation of isolated CNS blast cells in microculture. Nature 340:471-473. https://doi. org/10.1038/340471a0

31. Sulston JE, Schierenberg E, White JG, Thomson JN (1983) The embryonic cell lineage of the nematode Caenorhabditis elegans. Dev Biol 100:64-119. https://doi.org/10.1016/00121606(83)90201-4

32. Huang Q, Cohen MA, Alsina FC et al (2020) Intravital imaging of mouse embryos. Science (80-) 368:181-186. https://doi. org/10.1126/science.aba0210

33. Keizer K, Kuypers HGJM, Huisman AM, Dann O (1983) Diamidino yellow dihydrochloride (DY.2HCl); a new fluorescent retrograde neuronal tracer, which migrates only very slowly out of the cell. Exp Brain Res 51:179-191. https://doi. org/10.1007/BF00237193

34. Honig MG, Hume RI (1986) Fluorescent carbocyanine dyes allow living neurons of identified origin to be studied in long-term cultures. J Cell Biol 103:171-187. https://doi. org $/ 10.1083 /$ jcb.103.1.171

35. Honig MG, Hume RI (1989) Dil and DiO: versatile fluorescent dyes for neuronal labelling and pathway tracing. Trends Neurosci 12:333-341. https://doi.org/10.1016/0166-2236(89)90040 $-4$

36. Kornack DR, Rakic P (2001) The generation, migration, and differentiation of olfactory neurons in the adult primate brain. Proc Natl Acad Sci U S A 98:4752-4757. https://doi.org/10.1073/ pnas.081074998

37. Gould E, Vail N, Wagers M, Gross CG (2001) Adult-generated hippocampal and neocortical neurons in macaques have a transient existence. Proc Natl Acad Sci U S A 98:10910-10917. https ://doi.org/10.1073/pnas.181354698
38. Figueres-Oñate M, García-Marqués J, Pedraza M et al (2015) Spatiotemporal analyses of neural lineages after embryonic and postnatal progenitor targeting combining different reporters. Front Neurosci 9:1-11. https://doi.org/10.3389/fnins.2015.00087

39. Chalfie M, Tu Y, Euskirchen G et al (1994) Green fluorescent protein as a marker gene expression. Science (80-) 263:802-805

40. Son JH, Min N, Joh TH (1996) Early ontogeny of catecholaminergic cell lineage in brain and peripheral neurons monitored by tyrosine hydroxylase-lacZ transgene. Mol Brain Res 36:300-308. https://doi.org/10.1016/0169-328X(95)00255-Q

41. Holt CE, Garlick N, Cornel E (1990) Lipofection of cDNAs in the embryonic vertebrate central nervous system. Neuron 4:203214. https://doi.org/10.1016/0896-6273(90)90095-W

42. Itasaki N, Bel-Vialar S, Krumlauf R (1999) "Shocking" developments in chick embryology: electroporation and in ovo gene expression. Nat Cell Biol 1:E203-E207. https://doi. org/10.1038/70231

43. Luskin MB, Pearlman AL, Sanes JR (1988) Cell lineage in the cerebral cortex of the mouse studied in vivo and in vitro with a Recombinant Retrovirus. Neuron 1:635-647. https://doi. org/10.1016/0896-6273(88)90163-8

44. Levison SW, Chuang C, Abramson BJ, Goldman JE (1993) The migrational patterns and developmental fates of glial precursors in the rat subventricular zone are temporally regulated. Development 119:611-622

45. Noctor SC, Flint AC, Weissman TA et al (2001) Neurons derived from radial glial cells establish radial units in neocortex. Nature 409:714-720. https://doi.org/10.1038/35055553

46. Zerlin M, Milosevic A, Goldman JE (2004) Glial progenitors of the neonatal subventricular zone differentiate asynchronously, leading to spatial dispersion of glial clones and to the persistence of immature glia in the adult mammalian CNS. Dev Biol 270:200-213. https://doi.org/10.1016/j.ydbio.2004.02.024

47. Golden JA, Fields-Berry SC, Cepko CL (1995) Construction and characterization of a highly complex retroviral library for lineage analysis. Proc Natl Acad Sci U S A 92:5704-5708

48. Petit A, Legué E, Nicolas J (2005) Methods in clonal analysis and applications. Reprod nutr Dev 45:321-339. https://doi. org/10.1051/rnd

49. Sauer B (1987) Functional expression of the cre-lox site-specific recombination system in the yeast Saccharomyces cerevisiae. Mol Cell Biol 7:2087-2096. https://doi.org/10.1128/ MCB.7.6.2087.Updated

50. Golic KG, Lindquist S (1989) The FLP recombinase of yeast catalyzes site-specific recombination in the drosophila genome. Cell 59:499-509. https://doi.org/10.1016/0092-8674(89)90033 $-0$

51. Feil R, Wagner J, Metzger D, Chambon P (1997) Regulation of Cre recombinase activity by mutated estrogen receptor ligandbinding domains. Biochem Biophys Res Commun 237:752-757. https://doi.org/10.1006/bbrc.1997.7124

52. Dhaliwal J, Lagace DC (2011) Visualization and genetic manipulation of adult neurogenesis using transgenic mice. Eur J Neurosci 33:1025-1036. https://doi.org/10.1111/j.1460-9568.2011.07600 . $\mathrm{x}$

53. Lacar B, Young SZ, Platel J-C, Bordey A (2010) Imaging and recording subventricular zone progenitor cells in live tissue of postnatal mice. Front Neurosci 4:1-16. https://doi.org/10.3389/ fnins.2010.00043

54. Le Douarin N (2005) The Nogent Institute-50 years of embryology. Int J Dev Biol 49:85-103. https://doi.org/10.1387/ ijdb.041952nl

55. Lois C, Alvarez-Buylla A (1994) Long-distance neuronal migration in the adult mammalian brain. Science 264:1145-1148 
56. Merkle FT, Mirzadeh Z, Alvarez-Buylla A (2007) Mosaic organization of neural stem cells in the adult brain. Science 317:381384. https://doi.org/10.1126/science.1144914

57. Watt FM, Jensen KB (2009) Epidermal stem cell diversity and quiescence. EMBO Mol Med 1:260-267. https://doi.org/10.1002/ emmm.200900033

58. Feng G, Mellor RH, Bernstein M et al (2000) Imaging neuronal subsets in transgenic mice expressing multiple spectral variants of GFP. Neuron 28:41-51

59. Livet J, Weissman TA, Kang H et al (2007) Transgenic strategies for combinatorial expression of fluorescent proteins in the nervous system. Nature 450:56-62. https://doi.org/10.1038/natur e06293

60. Snippert HJ, van der Flier LG, Sato T et al (2010) Intestinal crypt homeostasis results from neutral competition between symmetrically dividing Lgr5 stem cells. Cell 143:134-144. https://doi. org/10.1016/j.cell.2010.09.016

61. Cai D, Cohen KB, Luo T et al (2013) Improved tools for the Brainbow toolbox. Nat Methods 10:540-547. https://doi. org/10.1038/nmeth.2450

62. Hadjieconomou D, Rotkopf S, Alexandre C et al (2011) Flybow: genetic multicolor cell labeling for neural circuit analysis in Drosophila melanogaster. Nat Methods 8:260-266. https://doi. org/10.1038/nmeth.1567

63. Hampel S, Chung P, McKellar CE et al (2011) Drosophila Brainbow: a recombinase-based fluorescence labeling technique to subdivide neural expression patterns. Nat Methods 8:253-259. https://doi.org/10.1038/nmeth.1566

64. Kanca O, Caussinus E, Denes AS et al (2014) Raeppli: a wholetissue labeling tool for live imaging of Drosophila development. Development 141:472-480. https://doi.org/10.1242/dev.102913

65. Pan YA, Livet J, Sanes JR et al (2011) Multicolor brainbow imaging in Zebrafish. Cold Spring Harb Protoc 6:1-8. https:// doi.org/10.1101/pdb.prot5546

66. Pan YA, Freundlich T, Weissman TA et al (2013) Zebrabow: multispectral cell labeling for cell tracing and lineage analysis in zebrafish. Development 140:2835-2846. https://doi.org/10.1242/ dev.094631

67. Veling MW, Li Y, Veling MT et al (2019) Identification of neuronal lineages in the drosophila peripheral nervous system with a "Digital" multi-spectral lineage tracing system. Cell Rep 29:3303-3312.e3. https://doi.org/10.1016/j.celrep.2019.10.124

68. Zong H, Espinosa JS, Su HH et al (2005) Mosaic analysis with double markers in mice. Cell 121:479-492. https://doi. org/10.1016/j.cell.2005.02.012

69. Hippenmeyer S, Johnson RL, Luo L (2013) Mosaic analysis with double markers reveals cell-type-specific paternal growth dominance. Cell Rep 3:960-967. https://doi.org/10.1016/j.celre p.2013.02.002

70. Griffin R, Sustar A, Bonvin M et al (2009) The twin spot generator for differential Drosophila lineage analysis. Nat Methods 6:600-602. https://doi.org/10.1038/nmeth.1349

71. Weber K, Bartsch U, Stocking C, Fehse B (2008) A multicolor panel of novel lentiviral "gene ontology" (LeGO) vectors for functional gene analysis. Mol Ther 16:698-706. https://doi. org $/ 10.1038 / \mathrm{mt} .2008 .6$

72. Weber K, Thomaschewski M, Warlich M et al (2011) RGB marking facilitates multicolor clonal cell tracking. Nat Med. https:// doi.org/10.1038/nm.2338

73. García-Marqués J, López-Mascaraque L (2013) Clonal identity determines astrocyte cortical heterogeneity. Cereb Cortex 23:1463-1472. https://doi.org/10.1093/cercor/bhs134

74. García-Moreno F, Vasistha NA, Begbie J, Molnár Z (2014) CLoNe is a new method to target single progenitors and study their progeny in mouse and chick. Development 141:1589-1598. https://doi.org/10.1242/dev.105254

75. Loulier K, Barry R, Mahou P et al (2014) Multiplex cell and lineage tracking with combinatorial labels. Neuron 81:505-520. https://doi.org/10.1016/j.neuron.2013.12.016

76. Kumamoto T, Maurinot F, Barry-Martinet R et al (2020) Direct readout of neural stem cell transgenesis with an integration-coupled gene expression switch. Neuron. https://doi.org/10.1016/j. neuron.2020.05.038

77. Garcia-Marques J, Espinosa-medina I, Ku K et al (2020) A programmable sequence of reporters for lineage analysis. Nat Neurosci. https://doi.org/10.1038/s41593-020-0676-9

78. Abdeladim L, Matho KS, Clavreul S et al (2019) Multicolor multiscale brain imaging with chromatic multiphoton serial microscopy. Nat Commun 10:1-14. https://doi.org/10.1038/ s41467-019-09552-9

79. Figueres-Oñate M, García-Marqués J, López-Mascaraque L (2016) UbC-StarTrack, a clonal method to target the entire progeny of individual progenitors. Sci Rep 6:33896. https://doi. org/10.1038/srep33896

80. Sánchez-González R, Bribián A, López-Mascaraque L (2020) Cell fate potential of NG2 progenitors. Sci Rep 10:1-12. https:// doi.org/10.1038/s41598-020-66753-9

81. Sánchez-González R, Figueres-Oñate M, Ojalvo-Sanz AC, López-Mascaraque L (2020) Cell progeny in the olfactory bulb after targeting specific progenitors with different UbC-startrack approaches. Genes (Basel). https://doi.org/10.3390/genes11030 305

82. Shapiro E, Biezuner T, Linnarsson S (2013) Single-cell sequencing-based technologies will revolutionize whole-organism science. Nat Rev Genet 14:618-630. https://doi.org/10.1038/nrg35 42

83. Fuentealba LC, Rompani SB, Parraguez JI et al (2015) Embryonic origin of postnatal neural stem cells. Cell 161:1644-1655. https://doi.org/10.1016/j.cell.2015.05.041

84. Mayer C, Jaglin XH, Cobbs LV et al (2015) Clonally Related forebrain interneurons disperse broadly across both functional areas and structural boundaries. Neuron 87:989-998. https://doi. org/10.1016/j.neuron.2015.07.011

85. Frieda KL, Linton JM, Hormoz S et al (2017) Synthetic recording and in situ readout of lineage information in single cells. Nature 541:107-111. https://doi.org/10.1038/nature20777

86. Kalhor R, Kalhor K, Mejia L et al (2018) Developmental barcoding of whole mouse via homing CRISPR. Science (80-). https:// doi.org/10.1126/science.aat 9804

87. Spanjaard B, Hu B, Mitic N et al (2018) Simultaneous lineage tracing and cell-type identification using CrIsPr-Cas9induced genetic scars. Nat Biotechnol 36:469-473. https://doi. org/10.1038/nbt.4124

88. McKenna A, Findlay GM, Gagnon JA et al (2016) Whole-organism lineage tracing by combinatorial and cumulative genome editing. Science (80-). https://doi.org/10.1126/science.aaf7907

89. Raj B, Wagner DE, McKenna A et al (2018) Simultaneous singlecell profiling of lineages and cell types in the vertebrate brain. Nat Biotechnol 36:442-450. https://doi.org/10.1038/nbt.4103

90. Chan MM, Smith ZD, Grosswendt S et al (2019) Molecular recording of mammalian embryogenesis. Nature 570:77-82. https://doi.org/10.1038/s41586-019-1184-5

91. Bowling S, Sritharan D, Osorio FG et al (2020) An engineered CRISPR-Cas9 mouse line for simultaneous readout of lineage histories and gene expression profiles in single cells. Cell. https ://doi.org/10.1016/j.cell.2020.04.048

92. Espinosa-Medina I, Garcia-Marques J, Cepko C, Lee T (2019) High-throughput dense reconstruction of cell lineages. Open Biol. https://doi.org/10.1098/rsob.190229 
93. Torii M, Hashimoto-Torii K, Levitt P, Rakic P (2009) Integration of neuronal clones in the radial cortical columns by EphA and ephrin-A signalling. Nature 461:524-528. https://doi. org/10.1038/nature08362

94. Brown KN, Chen S, Han Z et al (2011) Clonal production and organization of inhibitory interneurons in the neocortex. Science 334:480-486. https://doi.org/10.1126/science.1208884

95. Li Y, Lu H, Cheng P et al (2012) Clonally related visual cortical neurons show similar stimulus feature selectivity. Nature 486:118-121. https://doi.org/10.1038/nature11110

96. Bonaguidi MA, Wheeler MA, Shapiro JS et al (2011) In vivo clonal analysis reveals self-renewing and multipotent adult neural stem cell characteristics. Cell 145:1142-1155. https://doi. org/10.1016/j.cell.2011.05.024

97. Magavi S, Friedmann D, Banks G et al (2012) Coincident generation of pyramidal neurons and protoplasmic astrocytes in neocortical columns. J Neurosci 32:4762-4772. https://doi.org/10.1523/ JNEUROSCI.3560-11.2012

98. Álvarez-Aznar A, Martínez-Corral I, Daubel N et al (2020) Tamoxifen-independent recombination of reporter genes limits lineage tracing and mosaic analysis using CreERT2 lines. Transgenic Res 29:53-68. https://doi.org/10.1007/s11248-019-00177 $-8$

99. Beattie R, Postiglione MP, Burnett LE et al (2017) Mosaic analysis with double markers reveals distinct sequential functions of Lg11 in neural stem cells. Neuron 94:517-533.e3. https://doi. org/10.1016/j.neuron.2017.04.012

100. Mihalas AB, Hevner RF (2018) Clonal analysis reveals laminar fate multipotency and daughter cell apoptosis of mouse cortical intermediate progenitors. Dev. https://doi.org/10.1242/dev.16433 5

101. Pei W, Feyerabend TB, Rössler J et al (2017) Polylox barcoding reveals haematopoietic stem cell fates realized in vivo. Nature 548:456-460. https://doi.org/10.1038/nature23653

102. De Juan RC, Borrell V (2015) Coevolution of radial glial cells and the cerebral cortex. Glia 63:1303-1319. https://doi. org/10.1002/glia.22827

103. Lois C, Alvarez-Buylla A (1993) Proliferating subventricular zone cells in the adult mammalian forebrain can differentiate into neurons and glia. Proc Natl Acad Sci U S A 90:2074-2077. https://doi.org/10.1073/pnas.90.5.2074

104. Zhang Y, Liu G, Guo T et al (2020) Cortical neural stem cell lineage progression is regulated by extrinsic signaling molecule sonic hedgehog. Cell Rep 30:4490-4504.e4. https://doi. org/10.1016/j.celrep.2020.03.027

105. Redmond SA, Figueres-Oñate M, Obernier K et al (2019) Development of ependymal and postnatal neural stem cells and their origin from a common embryonic progenitor. Cell Rep 27:429441.e3. https://doi.org/10.1016/j.celrep.2019.01.088

106. Lake BB, Ai R, Kaeser GE et al (2016) Neuronal subtypes and diversity revealed by single-nucleus RNA sequencing of the human brain. Science (80-) 352:1586-1590. https://doi. org/10.1126/science.aaf1204

107. Loo L, Simon JM, Xing L et al (2019) Single-cell transcriptomic analysis of mouse neocortical development. Nat Commun 10:1-11. https://doi.org/10.1038/s41467-018-08079-9

108. Morel L, Men Y, Chiang MSR et al (2019) Intracortical astrocyte subpopulations defined by astrocyte reporter Mice in the adult brain. Glia 67:171-181. https://doi.org/10.1002/glia.23545

109. Batiuk MY, Martirosyan A, Wahis J et al (2020) Identification of region-specific astrocyte subtypes at single cell resolution. Nat Commun 11:1-15. https://doi.org/10.1038/s41467-019-14198-8

110. Marques S, van Bruggen D, Vanichkina DP et al (2018) Transcriptional convergence of oligodendrocyte lineage progenitors during development. Dev Cell 46:504-517.e7. https://doi. org/10.1016/j.devcel.2018.07.005
111. Marques S, Zeisel A, Codeluppi S et al (2016) Oligodendrocyte heterogeneity in the mouse juvenile and adult central nervous system. Science (80-) 352:1326-1329. https://doi.org/10.1126/ science.aaf6463

112. Bayraktar OA, Bartels T, Holmqvist S et al (2020) Astrocyte layers in the mammalian cerebral cortex revealed by a single-cell in situ transcriptomic map. Nat Neurosci 23:500-509. https://doi. org/10.1038/s41593-020-0602-1

113. Belgard TG, Marques AC, Oliver PL et al (2011) A transcriptomic atlas of mouse neocortical layers. Neuron 71:605-616. https://doi.org/10.1016/j.neuron.2011.06.039

114. Kanton S, Boyle MJ, He Z et al (2019) Organoid single-cell genomic atlas uncovers human-specific features of brain development. Nature 574(7778):418-422. https://doi.org/10.1038/s4158 6-019-1654-9

115. Bayraktar OA, Fuentealba LC, Alvarez-Buylla A, Rowitch DH (2015) Astrocyte development and heterogeneity. Cold Spring Harb Perspect Biol. https://doi.org/10.1101/cshperspect.a020362

116. Clavreul S, Abdeladim L, Hernández-Garzón E et al (2019) Cortical astrocytes develop in a plastic manner at both clonal and cellular levels. Nat Commun 10:1-14. https://doi.org/10.1038/ s41467-019-12791-5

117. Salipante SJ, Horwitz MS (2006) Phylogenetic fate mapping. Proc Natl Acad Sci U S A 103:5448-5453. https://doi. org/10.1073/pnas.0601265103

118. Wasserstrom A, Adar R, Shefer G et al (2008) Reconstruction of cell lineage trees in mice. PLoS ONE 3:e1939. https://doi. org/10.1371/journal.pone.0001939

119. Reeves MQ, Kandyba E, Harris S et al (2018) Multicolour lineage tracing reveals clonal dynamics of squamous carcinoma evolution from initiation to metastasis. Nat Cell Biol 20:699-709. https://doi.org/10.1038/s41556-018-0109-0

120. Tang YJ, Huang J, Tsushima $\mathrm{H}$ et al (2019) Tracing tumor evolution in sarcoma reveals clonal origin of advanced metastasis. Cell Rep 28:2837-2850.e5. https://doi.org/10.1016/j.celre p.2019.08.029

121. Martín-Pardillos A, Valls Chiva Á, Bande Vargas G et al (2019) The role of clonal communication and heterogeneity in breast cancer. BMC Cancer 19:1-26. https://doi.org/10.1186/s1288 5-019-5883-y

122. Davis JB, Krishna SS, Abi Jomaa R et al (2019) A new model isolates glioblastoma clonal interactions and reveals unexpected modes for regulating motility, proliferation, and drug resistance. Sci Rep 9:1-13. https://doi.org/10.1038/s41598-019-53850-7

123. Nato G, Caramello A, Trova $S$ et al (2015) Striatal astrocytes produce neuroblasts in an excitotoxic model of Huntington's disease. Development 142:840-845. https://doi.org/10.1242/dev.116657

124. Martín-López E, García-Marques J, Núñez-Llaves R, LópezMascaraque L (2013) Clonal astrocytic response to cortical injury. PLoS ONE 8:e74039. https://doi.org/10.1371/journ al.pone.0074039

125. Bribian A, Pérez-Cerdá F, Matute C, López-Mascaraque L (2018) Clonal glial response in a multiple sclerosis mouse model. Front Cell Neurosci 12:1-11. https://doi.org/10.3389/fncel.2018.00375

126. Gutiérrez Y, García-Marques J, Liu X et al (2019) Sibling astrocytes share preferential coupling via gap junctions. Glia 67:1852-1858. https://doi.org/10.1002/glia.23662

127. Picco N, Hippenmeyer S, Rodarte J et al (2019) A mathematical insight into cell labelling experiments for clonal analysis. J Anat 235:687-696. https://doi.org/10.1111/joa.13001

Publisher's Note Springer Nature remains neutral with regard to jurisdictional claims in published maps and institutional affiliations. 